\title{
PROCESS AND SYSTEMS Using computer simulation to model the expansion needs of the ambulatory emergency care unit at Derriford Hospital
}

\author{
Author: Daniel Chalk ${ }^{\mathrm{A}}$
}

Ambulatory emergency care (AEC) units can treat a wide variety of patients referred from the emergency department (ED) and acute general practitioners, helping to ease ED pressures and reduce unnecessary hospital admissions. We developed a simulation model to assess the potential benefits of expanding the size and opening times of the AEC unit at Derriford Hospital in Plymouth, both separately and in combination. The model predicted that an expanded size of AEC unit could help to improve the effectiveness of the unit, but not as much as extending the opening hours of the unit, which was predicted to result in a significant impact on the number of patients that can be seen and treated in the AEC unit. The hospital used the evidence generated by the model to support a business to case to extend opening hours of the AEC unit, and open the AEC unit at the weekend.

KEYWORDS: Operational research, urgent care, simulation modelling, discrete event simulation, ambulatory emergency care

\section{Introduction}

Units offering ambulatory emergency care (AEC) provide facilities that treat patients referred from acute general practitioners (GPs) and the emergency department (ED) who can be treated as outpatients and sent home the same day. ${ }^{1,2}$ The use of AEC units can be important for reducing the pressures on EDs, and avoiding unnecessary hospital admissions. ${ }^{1,3}$

Derriford Hospital in Plymouth is an acute hospital run by University Hospitals Plymouth NHS Trust. The hospital has a busy ED that sees around 90,000 new patients each year. ${ }^{4}$ The hospital currently runs an AEC unit that has proven to be successful, but which currently is only able to treat around 30-35 patients per day. ${ }^{1}$ The AEC unit sees and treats patients presenting with a range of conditions, including anaemia, some respiratory conditions, chest pain, abdominal pain and cellulitis, and can undertake transfusions and run clinics. The hospital was seeking to gain evidence to inform a decision about expanding the AEC unit, either in terms of physical space to see and treat

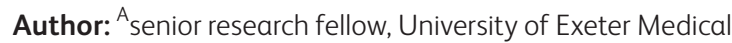
School, Exeter, UK more patients and/or in terms of extending the opening hours of the AEC unit.

The hospital approached us to develop a simulation model that could help them understand the optimal size and/or opening hours of the AEC unit to ensure that all patients who could be referred to the AEC unit could be seen and treated on the same day of arrival, and by close of service. Simulation modelling methods allow us to explore such 'what if' questions about a service by developing a model of the current system and then making changes in silico to predict the likely impact of proposed interventions. ${ }^{5,6}$ In this paper, we describe the simulation model that we developed, the evidence generated by the model, and how this evidence was used to inform decisions about expansion of the AEC unit.

\section{Methods}

We developed a discrete-event simulation model of Derriford Hospital's AEC unit using Simul8 model building software (Simul8 Corporation, Boston, USA). In the model, patients are referred into the $A E C$ unit from two sources - one representing the current level of AEC unit activity, and one representing potential AEC unit activity (which captures patients who could have been seen in the AEC unit but weren't, historically). Patients queue for the AEC unit, and when there is sufficient capacity for them to enter the unit they are seen and treated before being discharged. AEC unit attendance data was comprised of 3,659 anonymised patient records covering the whole of 2016. Data included the date and time of arrival into the AEC unit, the date and time of discharge, and various anonymised information about the patient, including their age, presenting condition, source of referral and discharge destination. We used the data to calculate times between arrivals into the AEC unit, which showed that the average inter-arrival time during the opening hours of the service was 39 minutes. Inter-arrival rates for current AEC unit activity in the model were sampled randomly to allow for variability, and represented using an exponential distribution with a mean of 39 minutes. Distributions are used in modelling to represent the pattern of variability of the real data. Models sample from distributions to represent the stochastic nature of real-world systems.

Potential AEC unit attendance data was drawn from two sources: one represented data from the wards in which patients had a 0 - or 1-day length of stay, who were deemed to have potentially been able to have been seen in an ambulatory capacity, while the 
other represented data from the ED and acute GPs. Both data sets included date and time of admission, which was used to estimate inter-arrival time for this potential activity. The ward data covered the whole of 2016, and inter-arrival times (in minutes) were fitted to a Weibull distribution, with $\alpha=0.982, \beta=101$ and minimum of 0 . The ED and acute GP data covered the period from 01 January 2015 to 30 November 2016, and inter-arrival times were fitted to an exponential distribution with a mean of 37.1 minutes.

The length of time patients spent in the AEC unit in the model was drawn from the 2016 AEC unit data. By calculating the difference between the discharge and arrival times, length of stay could be derived. The resultant lengths of stay were fitted to a gamma distribution, with $\alpha=2.63$ and $\beta=84.4$. AEC unit capacity in the model was defined as the number of patients that can be seen and treated simultaneously. This value was varied across the scenarios tested in the model.

The AEC unit in the model is open from $8 \mathrm{am}-8 \mathrm{pm}, 5$ days per week, emulating the opening times of the real AEC unit at the time of undertaking the project. The model runs for 1 day, and at the end of the day, it records the number of patients completed and discharged from the AEC unit. In the real AEC unit, any patients still in the unit towards closing time that are not able to be seen and treated are admitted to the medical assessment unit (MAU), and are counted as a hospital admission. Therefore, we also calculate the number of patients still in the AEC unit at the end of the day. Two-hundred and fifty runs of the simulation are undertaken for each scenario tested in the model, with average results taken across these runs. Multiple runs help to mitigate against runs of good or bad luck in systems which include elements of randomness. The model was used to answer a number of 'what if' questions about the potential expansion of the AEC unit, with appropriate reconfigurations of the model used to model each scenario.

> What if AEC unit capacity (to see and treat more patients simultaneously) was increased with existing opening hours in place? (Base case model parameterisation was used, with AEC unit capacities of 5, 10, 15 and 20 patients seen and treated simultaneously tested.)

> What if the AEC unit was open 24 hours per day, 7 days per week? (Model was set to run constantly for a period of 1 year, with average results per day calculated from this, with AEC unit capacities of 5, 10, 15 and 20 tested. Full 24-hour ED data was used to inform incoming activity for a 24 -hour scenario.)

$>$ If the AEC unit was open 7 days per week with current 8am-8pm opening hours, what would weekends look like? (Model interarrival times were parameterised using weekend arrival rates from potential activity only. Potential activity from ward arrivals was modelled using a Weibull distribution with $\alpha=1.04, \beta=116$ and minimum of 0 . Potential activity from ED and acute GPs was modelled using an exponential distribution with mean 42.9 minutes. AEC unit capacities of 5, 10, 15 and 20 patients were tested.)

> What if the AEC unit was open for the current 5 days per week, but for an additional 4 hours per day, during which no new arrivals are permitted, but patients already in the unit can be seen and treated? (Base case parameterisation was used, but with an additional 4 hours opening time, and a second distribution that generates zero arrivals from $8 \mathrm{pm}$ onwards. AEC unit capacities of 5, 10, 15 and 20 patients were tested.)

> What if the AEC unit was open for 7 days per week, and open for an additional 4 hours per day, during which no new arrivals are permitted, but patients already in the unit can be seen and treated - what would weekends look like? (Model was set up with inter-arrival rates as described in the third question, with opening times as described in previous question. AEC unit capacities of $5,10,15$ and 20 patients were tested.)

In testing these scenarios, we assumed that there were no limitations to staffing, and referral, diagnosis and treatment processes remained as they were in the current scenario. The results of the model were designed to inform potential staffing and process changes by demonstrating the potential impact of increased capacity and/or extended opening hours.

\section{Results}

Table 1 outlines the results from the 20 scenarios tested across the five 'what if' questions.

\section{What if AEC unit capacity was increased with existing opening hours in place?}

The model predicts that the capacity to see and treat 15 patients simultaneously would allow around 30 patients to be completed per day, with around 15 admitted to the MAU. Increasing capacity to 20 has only marginal benefit, with the model predicting just one more patient that could be completed, and one fewer admitted to the MAU. The hospital was not able to provide data to accurately specify their current capacity in the way this was captured by our model, but estimated that their current capacity was around 10 based on the outputs of the model at this capacity, suggesting that a $50 \%$ increase in capacity could lead to around five more patients being seen and treated in the AEC unit, and around five fewer admitted to the MAU.

\section{What if the AEC unit was open 24 hours per day, 7 days} per week?

The model predicts that a 24 hours per day, 7 days per week (24/7) AEC unit would need the capacity to see and treat at least 15 patients simultaneously in order to have anything approaching reasonable levels of time patients spend in the system. Even with a capacity of 20 , the model predicts that some patients could spend nearly a day in the system. A capacity of 10 is predicted to result in an average time in system of nearly 51 days, and a capacity of 5 would result in an average time in system of around 117 days. In reality, such times in system would not occur in a same-day see-and-treat service, and would instead contribute to increased admissions to the hospital. However, the model exposes the scale of the queue if it were to be unbounded.

\section{What would weekends look like if the AEC unit was} open 7 days per week with existing $8 \mathrm{am}-8 \mathrm{pm}$ opening hours?

The model predicts that AEC unit capacity requirements would be much lower at the weekends (assuming $8 \mathrm{am}-8 \mathrm{pm}$ opening hours), with limited additional benefit from having a capacity greater than 10, and even a capacity of five resulting in lower levels of patients admitted to the MAU than during the week. In the best scenario tested, 16 patients are completed and six patients are admitted to the MAU. 
Table 1. Overview of results from the 20 scenarios tested over the five 'what if' questions

\begin{tabular}{|c|c|c|c|}
\hline AEC capacity & Total completed & Number transferred to MAU & As $\%$ \\
\hline 5 & 13.52 & 31.15 & $69.73 \%$ \\
\hline 10 & 24.30 & 20.37 & $45.60 \%$ \\
\hline 15 & 29.58 & 15.09 & $33.78 \%$ \\
\hline 20 & 30.62 & 14.05 & $31.45 \%$ \\
\hline
\end{tabular}

What if the AEC unit was open 24 hours per day, 7 days per week?

$\begin{array}{llll}\text { AEC capacity } & \text { Average time in system, minutes } & \text { Minimum time in system, minutes } & \text { Maximum time in system, minutes } \\ 5 & 167866.01 & 89.11 & 335837.95 \\ 10 & 73181.54 & 56.79 & 146958.50 \\ 15 & 314.83 & 3.86 & 1560.07 \\ 20 & 224.26 & 2.65 & 1281.22\end{array}$

What would weekends look like if the AEC unit was open 7 days per week with existing $8 \mathrm{am}-8 \mathrm{pm}$ opening hours?

$\begin{array}{llll}\text { AEC capacity } & \text { Total completed } & \text { Number transferred to MAU } & \text { As \% } \\ 5 & 11.93 & 10.42 & 46.62 \% \\ 10 & 15.78 & 6.57 & 29.40 \% \\ 15 & 15.91 & 6.44 & 28.81 \% \\ 20 & 15.91 & 6.44 & 28.81 \%\end{array}$

What if the AEC unit was open 5 days a week, but for an additional 4 hours of seeing and treating patients (but no admissions)?

$\begin{array}{llll}\text { AEC capacity } & \text { Total completed } & \text { Number transferred to MAU } & \text { As \% } \\ 5 & 19.88 & 22.88 & 53.51 \% \\ 10 & 33.38 & 9.38 & 21.94 \% \\ 15 & 38.75 & 4.01 & 9.38 \% \\ 20 & 39.50 & 3.26 & 7.62 \%\end{array}$

What would weekends look like if the AEC unit was open 7 days a week with an additional 4 hours of seeing and treating patients (but no admissions)?

$\begin{array}{llll}\text { AEC capacity } & \text { Total completed } & \text { Number transferred to MAU } & \text { As \% } \\ 5 & 15.75 & 4.13 & 20.77 \% \\ 10 & 17.63 & 2.25 & 11.32 \% \\ 15 & 17.75 & 2.13 & 10.71 \% \\ 20 & 17.75 & 2.13 & 10.71 \%\end{array}$

'AEC capacity' represents the number of patients that can be seen and treated simultaneously in the AEC unit, and is used as a proxy for size of the AEC unit. 'Total completed' records the number of patients that were seen, treated and discharged from the AEC unit. 'Number transferred to MAU' represents the difference between the total number of patients arriving at the AEC unit, and the number completed, thereby estimating the number remaining at closure of the AEC unit that would be admitted to the MAU. 'As \%' represents the percentage of patients transferred to the MAU as a perccentage of the total the came into the AEC. For 'what if?' question 2, number completed and number transferred are no longer relevant, and instead we record the average, minimum and maximum time in system, including queuing for the $\mathrm{AEC}$ unit and time spent in the $\mathrm{AEC}$ unit. $\mathrm{AEC}$ = ambulatory emergency care; $\mathrm{MAU}$ = medical assessment unit.

What if the AEC unit was open 5 days a week, but for an additional 4 hours of seeing and treating patients (but no admissions)?

Comparing the results from this question with those from the first question, we see that extending the opening hours of the AEC unit by 4 hours (but not taking in any new patients during this time) is predicted to allow around six more patients to be seen and treated with a capacity of five, and around nine more to be seen and treated with a capacity of 10 or more. Around eight fewer patients would be transferred to the MAU with a capacity of five, and around 11 fewer with a capacity of 10 or more. Again, there are severely diminished returns of having an AEC unit capacity of more than 15 .

What would weekends look like if the AEC unit was open 7 days a week with additional 4 hours of seeing and treating patients (but no admissions)?

Comparing the results from this question with those from the previous question, we can see that, as with the third question, there is very limited benefit in having an AEC unit capacity greater 
Fig 1. Frequency of potential ambulatory emergency care unit activity by time of day in 2015 and 2016. Potential activity represents patients from the emergency department, referrals from acute general practitioners, and patients in wards with 0- or 1-day lengths of stay who it was deemed could have been seen and treated in the ambulatory emergency care unit if there had been capacity.

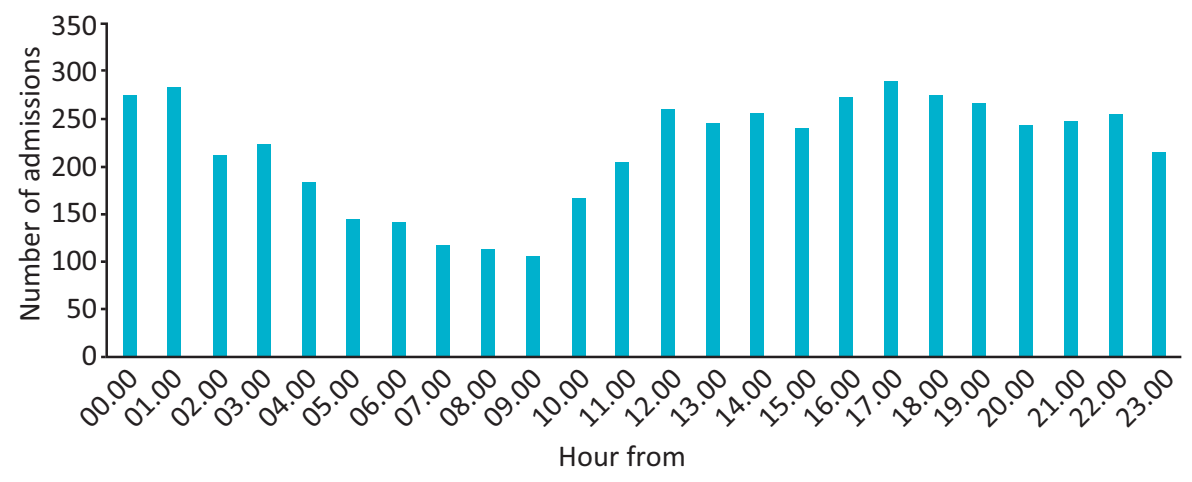

than 10 at the weekend. In this scenario, the difference between a capacity of five and 10 is even smaller, with a predicted 16 patients completed and four patients admitted to the MAU with a capacity of five, compared with around 18 patients completed and two patients admitted to the MAU with a capacity of 10 or more.

\section{Discussion}

The results from our modelling strongly suggest that there would only be limited benefit from expanding the physical size of the AEC unit alone at Derriford Hospital, and that much more significant benefits could be realised by extending the opening hours of the unit. In particular, the model predicts that simply extending the daily operation of the AEC unit by 4 hours (during which no new patients are taken in) could double the number of patients seen and treated within the AEC unit without needing to increase the size of the AEC unit. The model also suggests that a 24/7 AEC unit could see and treat all patients that could be seen in an ambulatory capacity (around 90 patients per day) but would need around a $50 \%$ increase in capacity to do so.

The predicted significant benefits of having extended opening hours occur principally because the potential flow of patients into the AEC unit from the emergency department occur over a 24-hour period, with peaks outside and towards the end of the $8 \mathrm{am}-8 \mathrm{pm}$ opening hours of the AEC unit (see Fig 1). Furthermore, the AEC unit is not open at weekends, leading to significant amounts of potential AEC unit activity needing to be dealt with in the ED at the weekends, potentially leading to large numbers of unnecessary hospital admissions. In addition, there is limited benefit in taking in new patients within the last 4 hours of operation of the AEC unit, because of the typical amount of time it takes for a patient to be seen and treated in the AEC unit, and because any patients not completed by the end of the AEC unit's operational hours are admitted to the MAU. This can be seen both in the real world data, in which incoming activity into the AEC unit drops sharply within the last 4 hours of operation (see Fig 2), and in the modelling results, which show the significant benefits of extending operational hours by 4 hours per day, even though such extensions would not see new patients taken in during this time.

If extending AEC unit opening into the weekends, the model suggests that the staffing requirements would not need to be as high as during the week. If the AEC unit was open for 12 hours on weekend days, the model predicts that only $66 \%$ of the weekday capacity would be needed to achieve the same levels of productivity, and that this would drop to $50 \%$ if the AEC unit was open for 16 hours. These results are encouraging when planning for staffing a service over the weekend, which can be more difficult. ${ }^{7}$

$A E C$ can be an effective way to treat patients outside of an inpatient setting and reduce burden on the acute hospital, and much work has been undertaken to help clinicians identify those patients who could be suitably treated in an ambulatory capacity. 8,9 Operational research approaches, such as the simulation modelling described in this paper, can further help to ensure that $A E C$ units are able to maximise the benefit for both patients and the health service. We would encourage other service improvement staff to consider using such methods to help inform the delivery and redesign of their AEC services.
Fig 2. Frequency of actual arrivals into ambulatory emergency care unit by time of day in 2016.

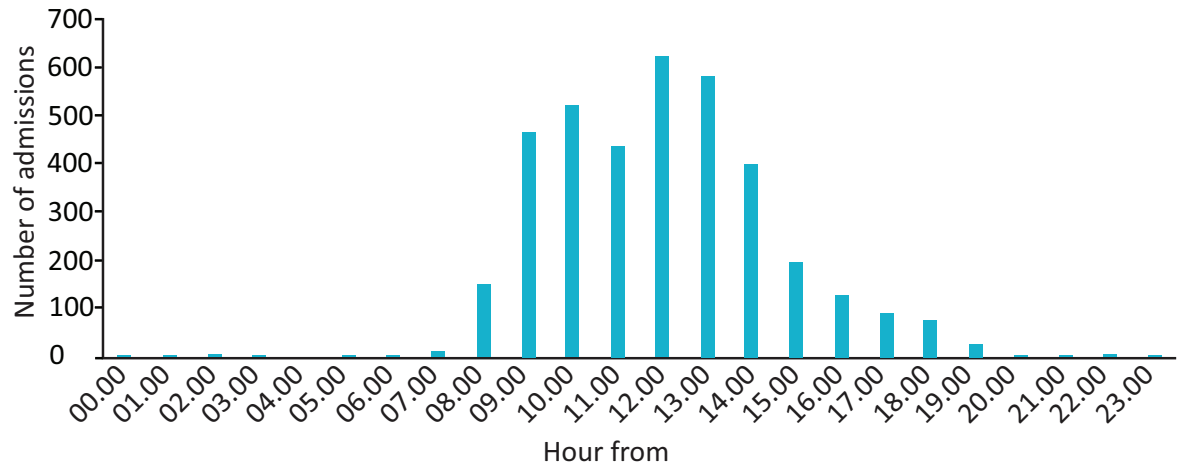




\section{Conclusion}

The results were presented to the executive board of Plymouth Hospitals NHS Trust (now University Hospitals Plymouth NHS Trust) in 2017. The evidence provided by this modelling work was used to inform a $£ 1$ million business case to extend the daily opening times of the AEC unit, to open the unit at the weekends, and to cover the additional staffing required for the extended opening times. We would encourage other acute trusts to use such modelling methods to assess how their AEC facilities might best benefit from expansion.

\section{Acknowledgements}

This report is independent research funded by the National Institute for Health Research Applied Research Collaboration South West Peninsula. The views expressed in this publication are those of the author and not necessarily those of the National Institute for Health Research or the Department of Health and Social Care. The author would like to thank Ryan Hunneman and Charlotte Edwards for their collaborative assistance on this project.

\section{References}

1 Carter A. The ambulatory care unit at Derriford Hospital. Clin Med 2014;14:250-4.

2 NHS Ambulatory Emergency Care Network. Directory of Ambulatory Emergency Care for Adults. AECN, 2018. www.ambulatoryemergencycare.org.uk/uploads/files/ 1/AEC-Directory \% 206th \% 20edition \% 20February \% 202018.pdf.
3 Strang G. The concept, delivery and future of medical ambulatory care. Clin Med 2008;8:276-9.

4 Ogilvie S, Hopgood K, Higginson I, Ives A, Smith JE. Why do parents use the emergency department for minor injury and illness? A cross-sectional questionnaire. JRSM Open 2016;7:205427041562369.

5 Rais A, Viana A. Operations Research in Healthcare: a survey. Int Trans Oper Res 2011;18:1-31.

6 Chalk D, Trent N, Vennam S, McGrane J, Mantle M. Reducing delays in the diagnosis and treatment of muscle-invasive bladder cancer using simulation modelling. J Clin Urol 2019;12:129-33.

7 Wyatt MG, Shearman C, Blair $P$ et al. Funding is insufficient for the NHS to work at weekend as it does in the week. BMJ 2013;346:f1854.

8 Hamad M, Connolly V. Ambulatory emergency care - improvement by design. Clin Med 2018;18:69-74.

9 Ala L, Mack J, Shaw R et al. Selecting ambulatory emergency care (AEC) patients from the medical emergency in-take: the derivation and validation of the Amb score. Clin Med 2012;12:420-6.

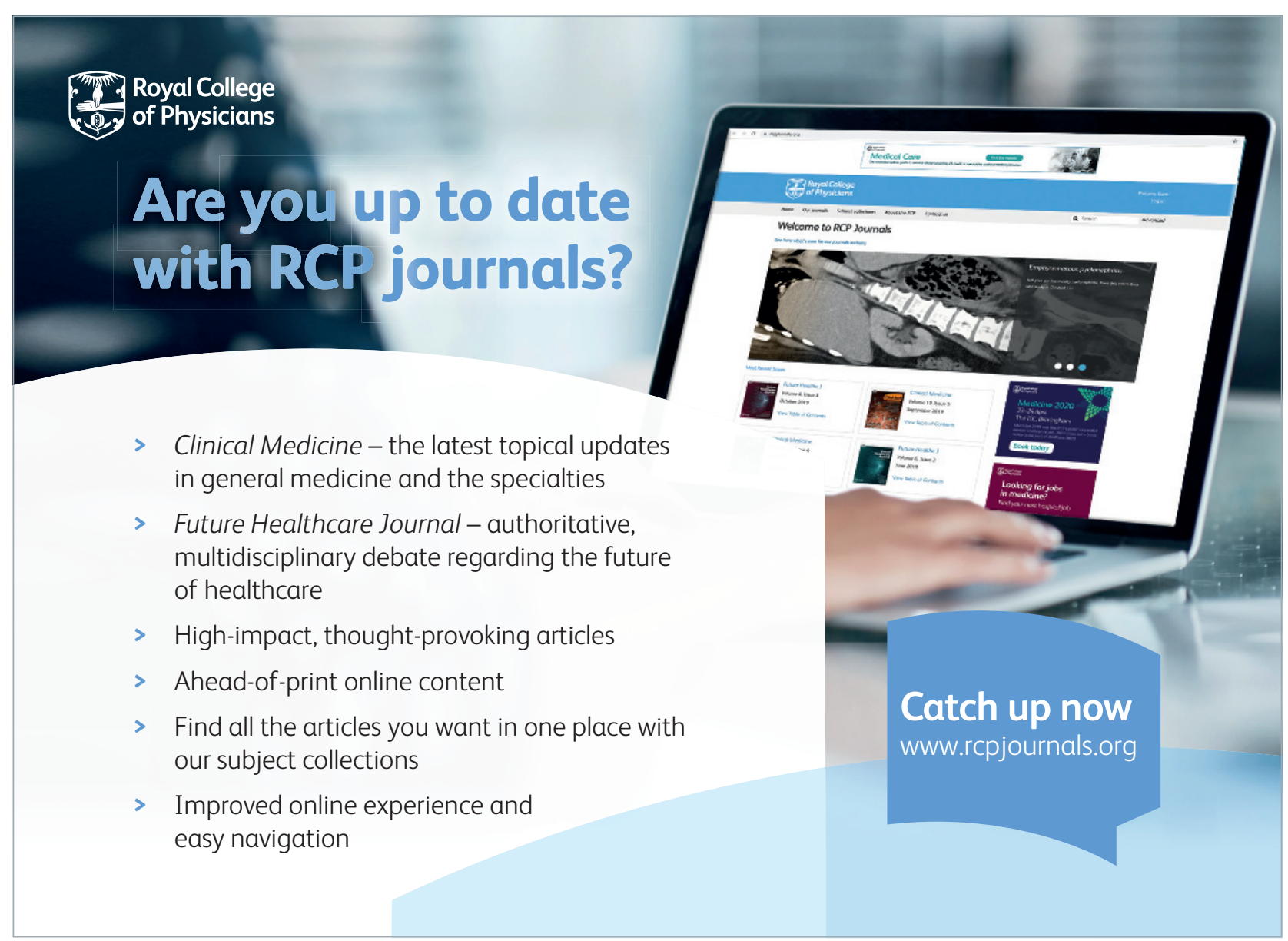
St Luke's Campus, Exeter EX1 1TX, UK.

Email:d.chalk@exeter.ac.uk 